\title{
Diagnostic de la contamination des eaux par les éléments traces métalliques dans la zone aurifère de Komabangou - Tillabéri, Niger
}

\author{
Abdourahamane TANKARI DAN-BADJO ${ }^{1 *}$, Didier Adamou TIDJANI ${ }^{1}$, \\ Tahar IDDER $^{2}$, Yadji GUERO ${ }^{1}$, Nomaou DAN LAMSO ${ }^{1}$, Ali MATSALLABI ${ }^{1}$, \\ Jean Marie Karimou AMBOUTA ${ }^{1}$, Cyril FEIDT $^{3}$, Thibault STERCKEMAN ${ }^{4}$ et \\ Guillaume ECHEVARRIA ${ }^{4}$ \\ ${ }^{I}$ Faculté d'Agronomie de Niamey, Université Abdou Moumouni de Niamey, Niger. \\ ${ }^{2}$ Laboratoire de Recherche sur la Phoeniciculture, Université KASDI Merbeh, 30000, Ouargla, Algérie. \\ ${ }^{3} U R$ AFPA, Université de Lorraine, INRA, France. \\ ${ }^{4}$ Laboratoire Sols et Environnement, Université de Lorraine, INRA, France. \\ *Auteur correspondant, E-mail: tankari@yahoo.fr
}

\section{RESUME}

A Komabangou, dans la région de Tillabéri au Niger, l'or est exploité, durant des décennies, de manière artisanale avec l'utilisation des méthodes inadéquates et des substances peu respectueuses de l'environnement comme le mercure et le cyanure. La présente étude a pour objectif de déterminer les teneurs en éléments traces métalliques (ETM) des eaux de Komabangou afin de prévoir les risques environnementaux et sanitaires liés à leur utilisation. Ainsi, des prélèvements d'eau ont été effectués sur 8 sites d'extraction d'or, 4 forages et 3 mares, et leurs concentrations en métaux tels que $\mathrm{As}, \mathrm{Cd}, \mathrm{Cu}, \mathrm{Hg}, \mathrm{Ni}, \mathrm{Pb}$ et $\mathrm{Zn}$ ont été analysées par spectrométrie de masse avec plasma à couplage inductif (ICP-MS). Les résultats obtenus révèlent une contamination importante de ces eaux et soulignent une variabilité significative des concentrations des ETM en fonction du métal analysé et du type d'eau. Dans la plupart des eaux, les valeurs limites réglementaires notamment celles de l'OMS ont été dépassées, cela pourrait poser des problèmes de toxicité pour les plantes, les animaux et la population locale. Par conséquent, la mise en place d'un programme de surveillance et de traitement des eaux contaminées de la zone de Komabangou serait nécessaire pour réduire les risques de contamination humaine.

(C) 2014 International Formulae Group. All rights reserved.

Mots clés : ETM, eaux, risque, site aurifère, Komabangou, Niger.

\section{INTRODUCTION}

Ces derniers temps, les problèmes posés par les contaminants chimiques, en particulier les éléments traces métalliques (ETM), dans l'environnement, suscitent l'intérêt de la communauté scientifique, car la protection de l'environnement passe par la connaissance du devenir de ces contaminants et de leurs effets sur les êtres vivants qui en dépendent. Leur présence dans les matrices environnementales est souvent occasionnée par les activités anthropiques notamment l'agriculture, l'élevage, l'industrie et le transport. Les humains peuvent être exposés aux ETM du fait de leur présence dans les sols et dans l'atmosphère ou à travers la 
consommation de denrées alimentaires ou d'eau contaminées (Tankari Dan Badjo et al., 2013). La contamination par les ETM a été tout d'abord mise en évidence dans les pays industriellement avancés en raison de leurs déversements industriels plus importants, et spécialement à la suite d'accidents dus à une pollution par le mercure $(\mathrm{Hg})$ et le cadmium (Hg) en Suède et au Japon (Goldberg, 1979). Dans quelques pays africains, les activités minières sont à l'origine d'importants apports des ETM dans l'environnement; citons par exemple $\mathrm{Hg}$ en Algérie, l'arsenic (As) en Namibie et en Afrique du Sud, l'étain (Sn) au Nigéria et en République Démocratique du Congo, et le cuivre $(\mathrm{Cu})$ en Zambie. Il ne fait pas de doute que les très fortes concentrations de certains métaux relevées dans des plans d'eau déterminés sont le résultat d'une pollution aiguë (Biney et al., 1994). Bien que le niveau des activités industrielles soit relativement moins élevé dans des régions moins développées comme l'Afrique, on y observe néanmoins une prise de conscience croissante de la nécessité de gérer rationnellement les ressources aquatiques et notamment de maîtriser les déversements de déchets dans l'environnement. Comme il est à prévoir que les activités industrielles et urbaines s'intensifieront dans toutes les régions du continent, cette question revêt désormais une importance encore plus grande.

En effet, plusieurs études ont été entreprises dans l'environnement aquatique afin d'évaluer la portée de ce phénomène. La plupart des études concernant les niveaux et la distribution des métaux lourds en Afrique ont surtout porté sur les zones urbaines et industrielles (Ahoussi et al., 2012 ; Taouil et al., 2012 ; Tankari Dan Badjo et al., 2013 ; Zegaoula et Khellaf, 2014). Selon les cas et la situation géographique, des dégâts importants à l'environnement peuvent être causés par les activités minières en libérant des quantités importantes de métaux. Les sources de contamination les plus importantes dans une activité minière sont les résidus de traitement, fins et riches en métaux (Brunel, 2005). Dans la zone aurifère de Komabangou, l'utilisation $\mathrm{du}$ cyanure et du mercure dans le processus d'extraction et de purification de l'or ainsi que la pression anthropique de la population des orpailleurs sur le milieu peuvent provoquer une pollution des eaux de surface et des eaux de la nappe phréatique et créer ainsi des problèmes environnementaux et sanitaires majeurs notamment sur la santé de la population, la faune et la flore.

Cette étude a pour objectif d'évaluer les teneurs en ETM des eaux de Komabangou afin de préciser les risques environnementaux et sanitaires liés à leur utilisation. Plus spécifiquement, il s'agit de (1) déterminer le degré de contamination des eaux par les ETM, (2) de les comparer aux valeurs normes réglementaires, et (3) de prévoir les risques liés à leur utilisation agricole ou leur consommation.

\section{MATERIEL ET METHODES \\ Description du site d'étude}

Située au Nord-Ouest de la ville de Niamey à environ $150 \mathrm{~km}$, la zone d'étude «Komabangou » localisée dans la région de Tillabéri est une vaste zone à forte densité d'indices et d'anomalies géochimiques aurifères. Se trouvant entre la latitude $14^{\circ} 01^{\prime} 41^{\prime \prime}$ 'et $14^{\circ} 07^{\prime} 56^{\prime}$ ' Nord et la longitude $01^{\circ} 02^{\prime} 12^{\prime}$ 'et $01^{\circ} 10^{\prime} 00^{\prime}$, Est, elle couvre une superficie de $157 \mathrm{~km}^{2}$ avec une population estimée à 36937 habitants répartis dans 18 villages du terroir (INS, 2010). C'est un site extrêmement riche en veines de quartz de plusieurs générations successives, dont certaines très riches en or, qui est exploité de manière traditionnelle. Les sites d'exploitation se présentent par une émergence sauvage et très désordonnée des puits et des carrières comme des galeries des bousiers. Sur ces sites, on observe des terrils de roches ou sables stériles rejetés lors de creusage des puits à la recherche de la roche utile contenant de l'or (Figure 1).

Les eaux utilisées pour la consommation humaine et aussi pour le processus d'extraction d'or proviennent à la fois des mares permanentes, puits et forages creusés sur les sites. La proximité de ces points d'eaux des sites d'exploitation aurifère et le déversement de rejets solides et effluents liquides dans la nature sans traitement préalable pourraient conduire à une contamination des eaux de surface et des eaux souterraines. 


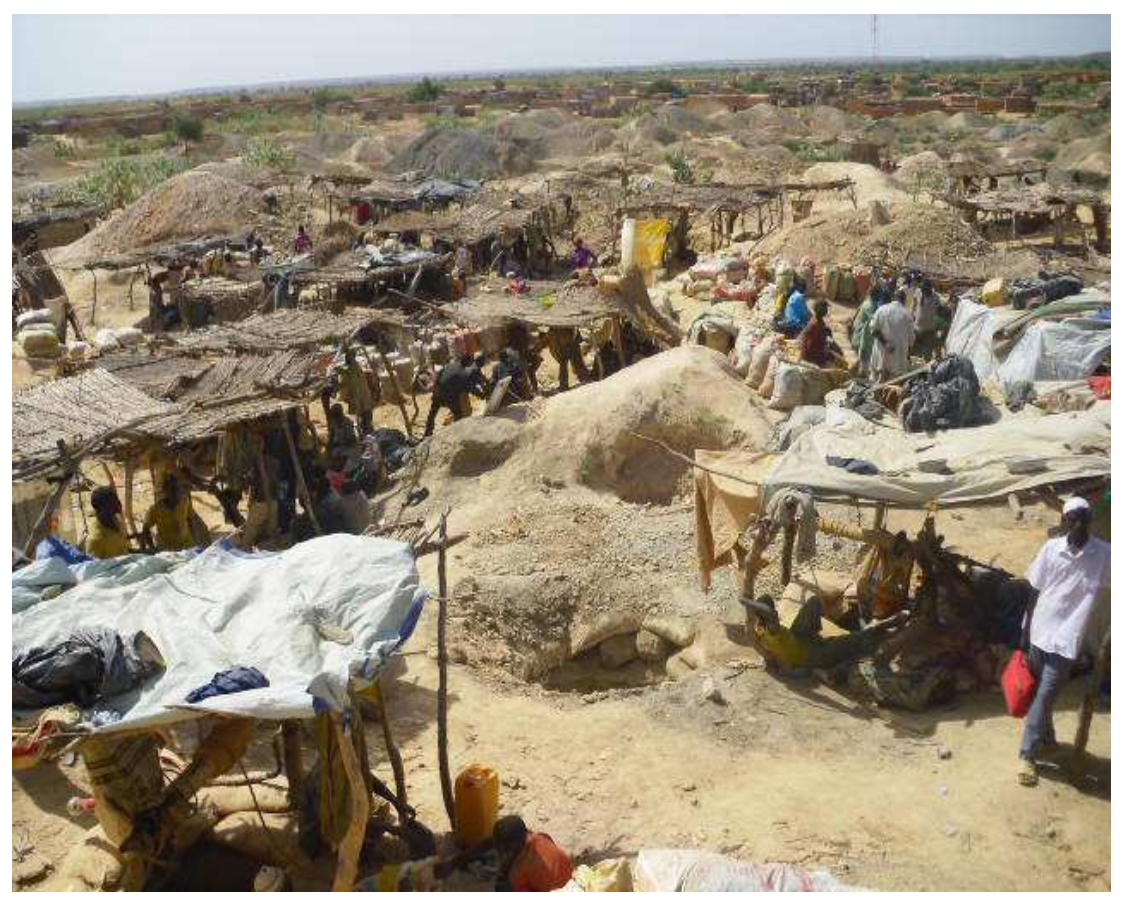

Figure 1: Vue d'un site aurifère de Komabangou.

\section{L'extraction et purification de l'or}

Dans la zone de Komabangou, deux principales méthodes d'extraction de l'or sur les différents sites d'orpaillage se distinguent : la séparation par écoulement gravitaire ou le procédé d'extraction par Sossoriya et le traitement par cyanuration.

\section{La technique de Sossoriya}

Le minerai rocheux préalablement concassé, broyé et bien tamisé, est mis en concentration dans l'eau, généralement dans des tonneaux. A travers un dispositif en plan incliné et en escalier recouvert d'un tapis, le concentré est passé par écoulement gravitaire afin d'assurer la séparation du sable et des particules d'or (Figure 2). Les particules, à cause de leur faible densité en comparaison avec les grains d'or, sont transportées par l'énergie hydraulique tandis que les pépites d'or restent piégées au niveau des barrettes sur le tapis. Le tapis est par la suite retiré et lavé dans des gamelles puis les grains d'or retenus sont libérés et recueillis dans la boue. Dans cette boue riche en or, on ajoute une petite quantité de mercure. La solution préparée est agitée afin de permettre au mercure d'attirer les particules qui sont dans la solution. On obtient ainsi un amas d'or qui sera ensuite lavé.

\section{Le traitement par cyanuration}

L'exploitation par cyanuration est effectuée dans des stations qui ont fait l'objet d'une autorisation administrative (permis d'exploitation) auprès des autorités compétentes du Ministère des Mines et de la Géologie étant donné que le cyanure est un produit très dangereux. Les produits utilisés sont : le cyanure de sodium $(\mathrm{CNNa})$; le zinc $(\mathrm{Zn})$; l'acide sulfurique $\left(\mathrm{H}_{2} \mathrm{SO}_{4}\right)$, l'acide nitrique $\left(\mathrm{H}_{2} \mathrm{NO}_{3}\right)$, le mercure $(\mathrm{Hg})$. Après avoir chargé les bassins par le rejet (sable) provenant des traitements classiques par Sossoriya, l'on verse un certain nombre de fûts d'eau (200 litres) et un gobelet de solution de cyanure $(200 \mathrm{ml})$ pour obtenir un mélange de terre cyanurée (Figure 3). Des tuyaux en PVC de petits diamètres en bas des bassins laissent passer l'eau filtrée de deux 
bassins dans un tube PVC en $\mathrm{U}$ de grand diamètre contenant des feuilles de zinc placé dans un $3^{\text {ème }}$ bassin cimenté situé entre les deux autres bassins. Les fragments d'or accompagnés d'impuretés sont retenus par les pastilles de zinc par oxydoréduction et l'excès d'eau cyanurée du tube est réutilisé pour reprendre le même processus ou rejeté. Suite à ces différentes réactions chimiques, les pastilles de zinc noircissent. Après trois jours, elles sont lavées avec de l'acide sulfurique et /ou de l'acide nitrique dilués dans des bassines en plastique. La solution résultant du lavage est laissée reposer jusqu'à décantation complète. Après transvasement de l'eau dans un autre récipient, le précipité obtenu est chauffé jusqu'à évaporation totale de l'eau pour obtenir l'or.

\section{Prélèvement des échantillons d'eau et analyse des éléments traces métalliques}

Pour connaître le degré de contamination des eaux par les ETM, des prélèvements d'échantillons d'eau ont été réalisés de manière à couvrir toute la zone aurifère de Komabangou. Le choix des points de prélèvement est basé sur la nature des eaux, la facilité d'accès et de prélèvement, et leur localisation par rapport aux rejets des polluants. En effet, ces prélèvements d'eau ont concerné : les eaux issues du processus d'exploitation de l'or, les eaux de surface (mares) et les eaux souterraines (puits et forages). Au niveau de chaque point de prélèvement, les échantillons d'eau destinés aux analyses chimiques ont été prélevés dans des bouteilles en polyéthylène d'une capacité de $250 \mathrm{ml}$, préalablement lavées à l'eau distillée. Sur le terrain, avant le remplissage des bouteilles, celles-ci ont été rincées trois fois, avec l'eau à prélever. Le remplissage des bouteilles a été fait à ras bord puis le bouchon vissé afin d'éviter tout échange gazeux avec l'atmosphère. Les échantillons d'eau ont été ensuite mis au frais et transportés au laboratoire pour les analyses chimiques. Au total, 15 échantillons d'eau (Tableau 1) ont été prélevés : 4 échantillons d'eaux usées provenant des sites de cyanuration; 4 échantillons des eaux du rejet de Sossoriya ; 4 échantillons d'eaux souterraines et 3 échantillons des eaux de surface.

Tableau 1: Localisation des points de prélèvement d'échantillons d'eau dans la zone de Komabangou.

\begin{tabular}{lcccc}
\hline $\mathbf{N}^{\circ}$ & Nature des eaux & \multicolumn{2}{c}{ Coordonnées géographiques } & Nom des Sites \\
\cline { 3 - 4 } & & Longitudes & Latitudes & \\
\hline 1 & & 001,06486 & 14,04992 & Mare de Guidan-Kwara \\
2 & Eau de surface & 001,09264 & 14,01370 & Mare de Kouhoun \\
3 & & 001,05043 & 14,0747 & Mare de Sounboul-Kongou \\
\hline 4 & & 001,05872 & 14,05836 & Forage de Bourah \\
5 & & 001,04997 & 14,071010 & Forage de Sounboul-Kongou \\
6 & Eau souterraine & 001,09283 & 14,01375 & Forage de Kouhoun \\
7 & & 001,05748 & 14,08591 & Puits (Nappe) site Abdallah \\
\hline 8 & Eau issue du procédé & 001,06077 & 14,08050 & Gawado-Komabangou \\
9 & d'extraction par & 001,06676 & 14,08561 & Kwascké-Komabangou \\
10 & Sossoriya & 001,06184 & 14,09320 & Nakiya-Komabangou \\
11 & & 001,05498 & 14,08849 & Dan-Dabino-Komabangou \\
\hline 12 & & 001,05163 & 14,10225 & Grillage Baba Djallo \\
13 & Eau issue du traitement & 001,06498 & 14,10192 & Grillage Moussa Morey \\
14 & par cyanuration. & 001,07366 & 14,08473 & Grillage Elh Saadou \\
15 & & 001,09436 & 14,08463 & Grillage Bidon \\
\hline
\end{tabular}


Après filtration, les concentrations en ETM (Al, As, Cd, Co, Cu, Fe, Hg, Mo, Mn, $\mathrm{Ni}, \mathrm{Pb}$ et $\mathrm{Zn}$ ) dans les eaux ont été déterminées par spectrométrie de masse avec plasma à couplage inductif (ICP-MS). Les teneurs en ETM sont exprimées en $\mathrm{mg} / \mathrm{l}$. Les limites de quantification sont de $0,058 \mathrm{mg} / \mathrm{L}$ pour l'Al, 0,0054 mg/l pour l'As, 0,0001 $\mathrm{mg} / \mathrm{L}$ pour le Cd et le Mo, 0,0013 mg/l pour le $\mathrm{Co}, 0,0019$ pour le $\mathrm{Cu}, 0,0027 \mathrm{mg} / \mathrm{l}$ pour le $\mathrm{Fe}$ et l'Hg. Elles sont de 0,0003, 0,0016, 0,0034 et $0,009 \mathrm{mg} / \mathrm{l}$ respectivement pour $\mathrm{Mn}, \mathrm{Ni}, \mathrm{Pb}$ et $\mathrm{Zn}$.

\section{Analyse statistique}

Les données obtenues ont été soumises à une analyse de variance pour identifier la présence ou non de différences significatives (au seuil de 5\% de probabilité) entre les concentrations moyennes des ETM en fonction de la nature des différents types d'eau. En présence de différences significatives, le test de Student Newman Keuls (au seuil de 5\%) a été effectué pour identifier les traitements significativement différents selon la variable considérée.

\section{RESULTATS}

Teneurs en éléments traces des eaux prélevées

Le Tableau 2 rassemble les résultats d'analyses des 12 éléments traces considérés dans les eaux prélevées sur le site de Komabangou. L'examen de ces données révèle la présence de plusieurs éléments chimiques à des teneurs variables en fonction de l'élément métallique considéré et de la nature des eaux. L'analyse statistique révèle que les concentrations en ETM sont significativement différentes en fonction de la nature des différents types d'eau.
Dans les eaux usées issues du traitement de l'or par cyanuration, les concentrations en ETM sont significativement plus élevées. Les teneurs moyennes sont de 1,$32 ; 0,32 ; 0,15 ; 24,29 ; 83,22$ et 108,47 $\mathrm{mg} / \mathrm{l}$ respectivement pour $\mathrm{Cd}, \mathrm{Co}, \mathrm{Mo}, \mathrm{As}, \mathrm{Hg}$ et $\mathrm{Mn}$. Des concentrations plus importantes allant jusqu'à $7859 \mathrm{mg} / \mathrm{l}$ ont été aussi déterminées dans les eaux de traitement par cyanuration. Les teneurs les plus fortes sont observées pour le Zn (7859 mg/l), suivi par ordre décroissant de l'Al (1458 mg/l), du Fe (697 mg/l) et du Cu (552 mg/l) (Tableau 2).

Les eaux usées provenant de la séparation de l'or par écoulement gravitaire communément appelées les eaux de Sossoriya présentent des teneurs moyennes en ETM significativement moins élevées comparées aux eaux de cyanuration. 10 éléments sur 12 présentent des teneurs moyennes inférieures à $1 \mathrm{mg} / \mathrm{l}$. Les $\mathrm{Zn}$ et le Mn présentent les teneurs les plus élevées avec respectivement 33,15 $\mathrm{mg} / \mathrm{l}$ et $1,60 \mathrm{mg} / \mathrm{l}$.

Les eaux de surface et les eaux souterraines prélevées dans la zone aurifère de Komabangou contiennent des teneurs en ETM très faibles comparativement aux eaux usées issues de Sossoriya ou de traitement par cyanuration. Les concentrations de $\mathrm{Ni}, \mathrm{Al}, \mathrm{As}$, $\mathrm{Fe}$ et $\mathrm{Zn}$ sont très faibles. Le $\mathrm{Pb}$ n'est pas détecté dans les eaux de surface alors qu'il se retrouve dans les eaux souterraines mais avec une teneur moyenne faible de l'ordre de 0,006 $\mathrm{mg} / \mathrm{L}$ (Tableau 2).

L'analyse comparative du Tableau 3 montre clairement que les eaux issues du traitement de l'or par cyanuration sont les plus contaminées par les ETM, suivies des eaux de Sossoriya; la contamination des eaux de surface semble équivalente à celle des eaux souterraines. 

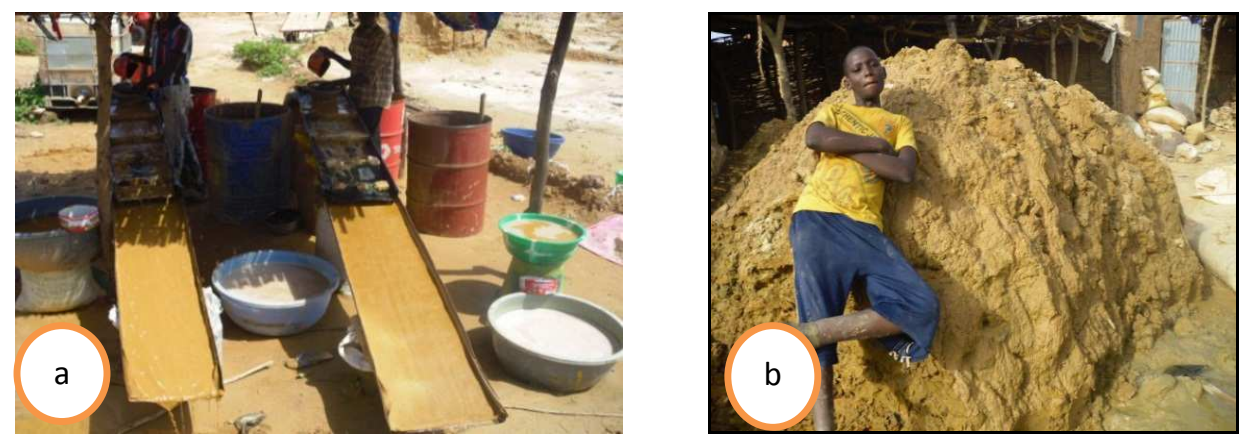

Figure 2 : Procédé d'extraction d'or par séparation gravitaire (a) et rejet du sable de lavage après extraction d'or (b).

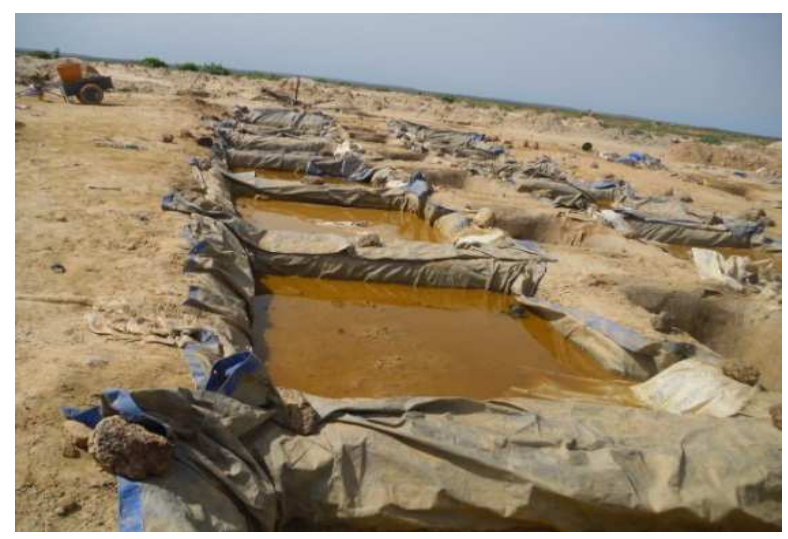

Figure 3 : Bassins de cyanuration utilisés dans la zone de Komabangou.

Tableau 2 : Teneurs moyennes des éléments traces métalliques dans les eaux de Komabangou $(\mathrm{mg} / \mathrm{L})$ selon la nature des eaux.

\begin{tabular}{lcccc}
\hline & Eau de cyanuration & Eau de Sossoriya & Eau de surface & Eau souterraine \\
\cline { 2 - 5 } & Moy $(\mathbf{n}=\mathbf{4})$. & Moy $(\mathbf{n}=\mathbf{4})$. & Moy $(\mathbf{n = 3})$. & Moy $(\mathbf{n}=\mathbf{4})$. \\
\hline $\mathrm{Al}$ & $1458,99 \mathrm{a}$ & $0,82 \mathrm{~b}$ & $0,049 \mathrm{c}$ & $0,132 \mathrm{bc}$ \\
$\mathrm{As}$ & $24,29 \mathrm{a}$ & $0,02 \mathrm{~b}$ & $0,006 \mathrm{c}$ & $0,019 \mathrm{~b}$ \\
$\mathrm{Cd}$ & $1,32 \mathrm{a}$ & $0,001 \mathrm{~b}$ & $0,0003 \mathrm{c}$ & $0,002 \mathrm{~b}$ \\
$\mathrm{Co}$ & $0,32 \mathrm{a}$ & $0,017 \mathrm{~b}$ & $0,001 \mathrm{c}$ & $0,002 \mathrm{c}$ \\
$\mathrm{Cu}$ & $552,69 \mathrm{a}$ & $0,04 \mathrm{c}$ & $0,038 \mathrm{c}$ & $0,275 \mathrm{~b}$ \\
$\mathrm{Fe}$ & $697,23 \mathrm{a}$ & $0,6 \mathrm{~b}$ & $0,025 \mathrm{c}$ & $0,052 \mathrm{c}$ \\
$\mathrm{Hg}$ & $83,22 \mathrm{a}$ & $0,38 \mathrm{~b}$ & $1,15 \mathrm{~b}$ & $1,393 \mathrm{~b}$ \\
$\mathrm{Mn}$ & $108,47 \mathrm{a}$ & $1,6 \mathrm{~b}$ & $0,055 \mathrm{c}$ & $0,098 \mathrm{c}$ \\
$\mathrm{Mo}$ & $0,15 \mathrm{a}$ & $0,02 \mathrm{a}$ & $0,005 \mathrm{~b}$ & $0,01 \mathrm{a}$ \\
$\mathrm{Ni}$ & $8,34 \mathrm{a}$ & $0,02 \mathrm{~b}$ & $0,007 \mathrm{c}$ & $0,019 \mathrm{~b}$ \\
$\mathrm{~Pb}$ & $3,878 \mathrm{a}$ & $0,012 \mathrm{~b}$ & $<\mathrm{LQ}$ & $0,006 \mathrm{c}$ \\
$\mathrm{Zn}$ & $7859,00 \mathrm{a}$ & $33,15 \mathrm{~b}$ & $6,21 \mathrm{c}$ & $42,18 \mathrm{~b}$ \\
\hline (a,b,c) $:$ Les concentrations moyennes affectées par la même lettre et sur la même ligne ne sont pas statistiquement \\
différentes au seuil de 5\%. LQ : Limite de quantification.
\end{tabular}


Tableau 3 : Comparaison des teneurs moyennes en éléments traces métalliques (ETM) des eaux de Komabangou (mg/l) avec les valeurs normes réglementaires.

\begin{tabular}{lcccccccc}
\hline & \multicolumn{2}{c}{ Eau de cyanuration } & \multicolumn{2}{c}{ Eau de Sossoriya } & \multicolumn{2}{c}{ Eau de surface } & \multicolumn{2}{c}{ Eau souterraine } \\
\cline { 2 - 9 } & & VN* $^{*}$ & \multicolumn{3}{c}{ VN* $^{*}$} & $\cdot$ & VN** $^{*}$ & VN** \\
\hline $\mathrm{Al}$ & 1458,99 & - & 0,82 & - & 0,049 & 0,2 & 0,132 & 0,2 \\
$\mathrm{As}$ & 24,29 & 0,1 & 0,02 & 0,1 & 0,006 & 0,01 & 0,019 & 0,01 \\
$\mathrm{Cd}$ & 1,32 & 0,02 & 0,001 & 0,02 & 0,0003 & 0,003 & 0,002 & 0,003 \\
$\mathrm{Co}$ & 0,32 & 1 & 0,017 & 1 & 0,001 & & 0,002 & \\
$\mathrm{Cu}$ & 552,69 & 1 & 0,04 & 1 & 0,038 & 2 & 0,275 & 2 \\
$\mathrm{Fe}$ & 697,23 & - & 0,6 & - & 0,025 & 0,3 & 0,052 & 0,3 \\
$\mathrm{Hg}$ & 83,22 & 0,5 & 0,38 & 0,5 & 1,15 & 0,01 & 1,393 & 0,01 \\
$\mathrm{Mn}$ & 108,47 & - & 1,6 & - & 0,055 & 0,4 & 0,098 & 0,4 \\
$\mathrm{Mo}$ & 0,15 & - & 0,02 & - & 0,005 & 0,07 & 0,01 & 0,07 \\
$\mathrm{Ni}$ & 8,34 & 1 & 0,02 & 1 & 0,007 & 0,07 & 0,019 & 0,07 \\
$\mathrm{~Pb}$ & 3,87 & 0,5 & 0,012 & 0,5 & $<\mathrm{LQ}$ & 0,01 & 0,006 & 0,01 \\
$\mathrm{Zn}$ & 7859 & 1 & 33 & 1 & 6,21 & 3 & 42,18 & 3 \\
\hline
\end{tabular}

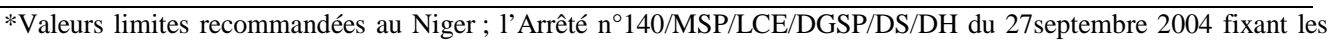
normes de rejet des déchets dans le milieu naturel ; **Normes OMS, Directives 2006 de l'Organisation Mondiale de la Santé sur la qualité de l'eau de boisson en ce qui concerne la sécurité en matière de l'eau potable (OMS, 2008); LQ : Limite de quantification.

\section{DISCUSSION}

Les fortes teneurs en $\mathrm{Al}, \mathrm{Cu}, \mathrm{Fe}$ et $\mathrm{Mn}$ retrouvées dans les eaux cyanurées de Komabangou ne sont pas surprenantes car ces éléments sont abondants dans les minerais d'or et dans les sols (Smouni et al., 2010; Tankari Dan Badjo et al., 2013) ; et l'attaque acide du minerai lors du processus d'extraction d'or permet leur minéralisation et leur solubilisation dans ces eaux. En effet, la solubilité de l'Al et du Mn est fortement affectée par le $\mathrm{pH}$ acide du fait de l'utilisation de l'acide cyanurique et de l'acide nitrique lors du traitement par cyanuration. $\mathrm{La}$ contamination par l'As est probablement liée à l'extraction du minerai car l'As est un composant important du minerai d'or. Pour le $\mathrm{Zn}$, la contamination spécifique est due à l'utilisation de pastilles de $\mathrm{Zn}$ dans le procédé de traitement pour la récupération de l'or à partir de solutions acides de lixiviation des minerais. Le mercure est également utilisé dans le procédé de purification de l'or et sa concentration dans les 4 types d'eau de Komabangou est très élevée. Du fait de sa volatilité et de sa solubilité, il peut facilement atteindre les cours d'eau et les nappes. Pour les autres éléments $(\mathrm{Cd}, \mathrm{Pb}, \mathrm{Ni})$, ils sont probablement associés à une contamination par les éléments traces des pastilles de zinc.
En comparant les teneurs en ETM dans les eaux de surface et souterraine de Komabangou avec celles des autres études, il ressort que les concentrations de $\mathrm{Cd}, \mathrm{Cu}$, Ni et $\mathrm{Pb}$ sont assez proches de celles détectées dans les eaux de l'oued Tislit-Talsint au Maroc oriental (Taouil et al., 2012), variant de 0,003 à $0,006 \mathrm{mg} / \mathrm{L} ; 0,004$ à $0,012 \mathrm{mg} / \mathrm{L} ; 0,025$ à $0,053 \mathrm{mg} / \mathrm{L}$ et de 0,015 à $0,079 \mathrm{mg} / \mathrm{L}$ respectivement. Par contre, les teneurs en ETM détectées dans ces eaux de Komabangou sont largement supérieures à celles retrouvées dans les eaux de la seine en France (Raven et Leoppert, 1997).

En vue de déterminer les risques liés à l'utilisation agricole et/ou la consommation des eaux de Komabangou, les teneurs en ETM de ces eaux ont été comparées avec les valeurs normatives (Tableau 3). L'examen de ces données et leur confrontation à titre comparatif avec les valeurs limites de l'organisation mondiale de la santé (OMS, 2008) sur la qualité de l'eau de boisson (eau souterraine et eau de surface); et les normes nigériennes relatives aux valeurs limites fixant les rejets des déchets liquides dans le milieu 
naturel, permettent de dégager les principales considérations suivantes :

- dans les eaux de cyanuration, les concentrations moyennes de $\mathrm{As}, \mathrm{Cd}, \mathrm{Cu}, \mathrm{Hg}$, $\mathrm{Ni}, \mathrm{Pb}$ et $\mathrm{Zn}$ sont supérieures aux valeurs limites nigériennes de rejet des eaux usées dans le milieu naturel. Pour le $\mathrm{Zn}$, ce dépassement est exceptionnel, de l'ordre de milliers de fois. Pour l'Al, le Fe, le $\mathrm{Mn}$, et le Mo, la norme nigérienne ne prévoit pas des valeurs limites. Cependant, les teneurs de ces éléments détectés dans les eaux de cyanuration sont supérieures aux concentrations maximales recommandées pour les eaux d'irrigation (Pescod, 1992). Le déversement de ces eaux sans traitement dans la nature pourrait causer des risques environnementaux et sanitaires.

- pour les eaux usées de Sossoriya, le dépassement des valeurs normatives concerne uniquement les éléments chimiques suivants : As et $\mathrm{Zn}$ qui dépassent de 2 et 33 fois les valeurs limites de rejet des eaux usées fixées par le Niger pour ces éléments à $0,1 \mathrm{mg} / \mathrm{L}$ et 1 $\mathrm{mg} / \mathrm{L}$ respectivement. Toute utilisation de ces eaux devrait donc être interdite avant une décontamination en As et en $\mathrm{Zn}$.

- dans les eaux de surface et les eaux souterraines de Komabangou, on observe un dépassement des valeurs limites préconisées par l'OMS pour le $\mathrm{Zn}$ et l'Hg. $\mathrm{La}$ consommation de ces eaux par l'homme et les animaux pourrait donc engendrer à moyen et long termes des conséquences sanitaires.

La mauvaise gestion des eaux usées de cyanuration de Komabangou riches en $\mathrm{As}, \mathrm{Cu}$, $\mathrm{Ni}$ et $\mathrm{Zn}$ pourrait engendrer des conséquences environnementales car ces éléments sont très toxiques pour les végétaux et, présentent de ce fait un danger pour la chaîne alimentaire. Le déversement de ces eaux dans le milieu naturel va engendrer une contamination du sol, et par infiltration une possible pollution de la nappe phréatique, ou par ruissellement une pollution des eaux de surface. Les végétaux qui se développent sur les sols pollués ou qui sont irrigués avec ces eaux chargées en métaux pourraient à leur tour se contaminer et engendrer de risque tout au long de la chaîne alimentaire.

L'As, le $\mathrm{Cd}, \mathrm{l}$ 'Hg et le $\mathrm{Pb}$ sont très toxiques pour l'Homme; leur détection dans les eaux de surface et les eaux souterraines de Komabangou à des teneurs supérieures aux limites autorisées est source d'inquiétude car ces eaux sont utilisées pour la consommation humaine et animale. Chez l'Homme, l'accumulation d'ETM dans l'organisme peut conduire à des dysfonctionnements du système rénal $(\mathrm{Pb}, \mathrm{Cd})$, neurologique $(\mathrm{Pb}, \mathrm{As}$, $\mathrm{Cd}, \mathrm{Mn})$ ou hépatique $(\mathrm{Cd})$ et provoquer des cancers (Goyer et Clarkson, 2001).

\section{Conclusion}

L'étude de la contamination par les ETM dans la zone aurifère de Komabangou a permis de mettre en évidence la présence de plusieurs métaux à différents niveaux dans les eaux de cette localité. Cette pollution des eaux semble avoir pour origine anthropique les procédés d'extraction d'or par cyanuration et la séparation par gravimétrie, et pour origine naturelle, la roche mère (le minerai d'or) riche en certains éléments comme l'As. D'une manière générale, les eaux issues de procédés d'extraction d'or sont les plus contaminées suivies des eaux de surface et des eaux souterraines. La comparaison des teneurs en ETM dans ces eaux avec les valeurs limites réglementaires a révélé un dépassement des valeurs limites nigériennes de rejet des eaux usées dans le milieu naturel, en As, Cd, Co, $\mathrm{Cu}, \mathrm{Hg}, \mathrm{Ni}, \mathrm{Pb}$ et $\mathrm{Zn}$ pour les eaux de cyanuration, et en As et $\mathrm{Zn}$ pour les eaux de Sossoriya. Dans les eaux souterraines et les eaux de surface, les résultats obtenus ont montré la présence de certains ETM tels que le $\mathrm{Zn}$ et l'Hg à des concentrations supérieures aux valeurs limites recommandées par l'OMS pour la qualité de l'eau de boisson. L'utilisation de ces eaux sans traitement préalable pourrait donc engendrer des risques environnementaux et sanitaires. En vue d'améliorer la qualité de ces eaux et de réduire voire d'éliminer ces risques potentiels, la mise en place d'un programme de 
surveillance et de traitement de ces eaux contaminées s'avère nécessaire.

\section{REMERCIEMENTS}

Les auteurs remercient l'UEMOA et le projet SASACID d'ANAFE pour l'appui financier qu'ils ont apporté pour la réalisation de cette étude.

\section{REFERENCES}

Ahoussi KE, Koffi YB, Loko S, Kouassi AM, Soro G, Biemi J. 2012. Caractérisation des éléments traces métalliques $(\mathrm{Mn}, \mathrm{Ni}, \mathrm{Zn}$, $\mathrm{Cd}, \mathrm{Cu}, \mathrm{Pb}, \mathrm{Cr}, \mathrm{Co}, \mathrm{Hg}, \mathrm{As}$ ) dans les eaux superficielles de la commune de Marcory, Abidjan Côte d'Ivoire : cas du village d'Abia Koumassi. Geo-Eco-Trop., 36: 159-174.

Biney C, Amuzu AT, Calamari D, Kaba N, Mbome IL, Naeve H, Ochumba O, Osibanjo O, Radegonde V, Saad MAH. 1994. Etude des métaux lourds, Revue de la Pollution dans l'Environnement Aquatique Africain, FAO, 25: 1-6.

Brunel C. 2005. Dynamique des éléments traces métalliques $(\mathrm{Pb}, \mathrm{Zn}$ et $\mathrm{Cd})$ sur un petit bassin versant contaminé par des déchets miniers-cas du bassin versant amont du Lez (Ariège, Pyrénées). Ph.D thesis, Toulouse III University, France, $276 \mathrm{p}$.

Goldberg ED. 1976. La Santé des Océans. UNESCO : Paris; 188.

Goyer RA, Clarkson TW. 2001. Toxic effects of metals. In Toxicology: The Basic Science of Poisons, ( $6^{\text {th }}$ edn), Klassen CD (ed). Casarett \& Doull's. McGraw-Hill: New York, NY, USA; 811-. 837.

INS. 2010. Institut National de la Statistique, Rapport de l'estimation de la population du Niger, 30p.

OMS (World Health Organization). 2008. Guidelines for Drinking Water Quality,
Recommendations (vol.1, $2^{\text {nd }}$ edn). WHO: Geneva; 130.

Pescod MB. 1992. Wastewater treatment and use in agriculture. Publication FAO, Irrigation \& Drainage, 47: 117.

Raven KP, Leoppert RH. 1997. Heavy metals in the environment "Trace element composition of fertilizers and soil Amendment". J. Enviro. Qual., 26: 551557.

Smouni A, Ater M, Auguy F, Laplaze L, Mzibri ME, Berhada F, Filali-Maltouf A, Doumas P. 2010. Evaluation de la contamination par les éléments traces métalliques dans une zone minière du Maroc oriental. Cah. Agric., 19(4): 273279.

Tankari Dan-Badjo A, Yadji G, Nomaou DL, Moussa Tawaye O. 2012. Risque d'exposition de la population de Niamey (Niger) aux métaux lourds à travers la consommation des produits maraichers. Revue des BioRessources, 2(2): 88-99.

Tankari Dan-Badjo A, Guero Y, Dan Lamso N, Tidjani AD, Ambouta KJM, Feidt C, Sterckeman T, Echevarria G. 2013. Evaluation de la contamination des sols par les éléments traces métalliques dans les zones urbaines et périurbaines de la ville de Niamey (Niger). Revue des BioRessources, 2(2): 82-95.

Taouil H, Ben Ahmed S, Hajjaji N, Srhiri A. 2012. Plomb, cadmium, cuivre et nickel dans le bassin versant de Guir; impact sur la qualité des eaux de surface d'oued Tislit-Talsint (Maroc Oriental). Science Lib Editions Mersenne, 4 : $\mathrm{N}^{\circ} 120111$.

Zegaoula W, Khellaf N. 2014. Evaluation du degré de pollution des rejets liquides et atmosphériques du complexe fertial Annaba (Algérie). Larhyss Journal, 18: 77-91. 